\title{
Impact of Mid-Luteal Phase GnRH Agonist Administration on Reproductive Outcomes in GnRH Agonist-Triggered Cycles: A Randomized Controlled Trial
}

\author{
Abdelhamid Benmachiche ${ }^{*}$, Sebti Benbouhedja ${ }^{1}$, Abdelali Zoghmar $^{1}$, Amel Boularak ${ }^{1}$ \\ and Peter Humaidan ${ }^{2,3}$ \\ ${ }^{1}$ Center for Reproductive Medicine, Clinique Ibn Rochd, Constantine, Algeria, ${ }^{2}$ The Fertility Clinic, Skive Regional Hospital, \\ Skive, Denmark, ${ }^{3}$ Faculty of Health Aarhus University, Aarhus, Denmark
}

Objective: To explore whether the addition of a mid-luteal bolus of $\mathrm{GnRH}$ agonist $(\mathrm{GnRHa})$ improves the implantation rate (IR) in in vitro fertilization (IVF) cycles.

Design: A randomized controlled trial.

\section{OPEN ACCESS Setting: Private IVF center.}

Edited by: Ren-Shan Ge, Wenzhou Medical University, China

Reviewed by:

Shahar Kol, Rambam Health Care

Campus, Israel

Carlos Dosouto,

Dexeus Women's Health, Spain

${ }^{*}$ Correspondence: Abdelhamid Benmachiche benmachiche@gmail.com

Specialty section: This article was submitted

to Reproduction,

a section of the journal Frontiers in Endocrinology

Received: 21 March 2017 Accepted: 22 May 2017

Published: 15 June 2017

Citation: Benmachiche A, Benbouhedja S,

Zoghmar A, Boularak A and

Humaidan P (2017) Impact of Mid-Luteal Phase GnRH Agonist Administration on Reproductive Outcomes in GnRH Agonist-Triggered Cycles: A Randomized Controlled Trial. Front. Endocrinol. 8:124. doi: 10.3389/fendo.2017.00124
Patients: 328 IVF/intracytoplasmic sperm injection patients were triggered with $\mathrm{GnRHa}$ and received 1,500 IU HCG on the day of oocyte pick-up (OPU) in addition to a standard luteal phase support (LPS).

Intervention(s): In addition, the study group received a bolus of GnRHa 6 days after OPU, whereas the control group did not.

Main outcome measure: Implantation rate.

Secondary outcome measure(s): Ongoing pregnancy (OP) and live birth (LB) rates.

Results: Although serum concentrations of $\mathrm{FSH}, \mathrm{LH}, \mathrm{E} 2$, and $\mathrm{P}$ on day OPU +7 were significantly higher in the study group compared to the control group, the IR was not statistically different between the treatment group (27\%) and the control group (23\%) [odds ratio (OR) $1.2(95 \% \mathrm{Cl} 0.9-1.7), P<0.27$ ]. Similarly, the OP rate was $37 \%$ in the treatment group and 31\% in the control group [OR 1.3 (95\% Cl 0.8-2.0), $P<0.23$ ]. The $\mathrm{LB}$ rate was $36 \%$ in the treatment group and $31 \%$ in the control group [OR: $1.3(95 \% \mathrm{Cl}$ 0.8-2.0), $P<0.27$ ].

Conclusion: Although a trend toward a higher IR and pregnancy rate was observed in the treatment group, this difference was not statistically significant. However, the absolute risk difference of $5 \%$ found for LB is clinically relevant, warranting further investigation.

NCT: 02053779.

Keywords: GnRHa trigger, in vitro fertilization, luteal phase support, luteal GnRH agonist, mid-luteal steroids

Abbreviations: GnRHa, GnRH agonist; IVF, in vitro fertilization; ICSI, intracytoplasmic sperm injection; FSH, folliclestimulating hormone; LH, luteinizing hormone; HCG, human chorionic gonadotropin; LPS, luteal phase support; IR, implantation rate; OP, ongoing pregnancy; LB, live birth; OHSS, ovarian hyperstimulation syndrome; E2, estradiol; P, progesterone; CIs, confidence intervals; AUC, the area under the receiver operating characteristic (ROC) curve. 


\section{INTRODUCTION}

Initial studies in in vitro fertilization (IVF)/intracytoplasmic sperm injection (ICSI) patients have demonstrated that the use of a GnRH agonist (GnRHa) trigger, followed by fresh transfer and a standard luteal phase support (LPS) was associated with unacceptably high rates of loss in early pregnancy compared to hCG trigger, particularly in normal responder (NR) patients $(1,2)$. It has been subsequently concluded that this early pregnancy loss was caused by luteal phase (LP) insufficiency, despite the use of a standard LPS package of progesterone (P) and estradiol (E2). The LP defect was primarily caused by reduced early-mid-luteal luteinizing hormone $(\mathrm{LH})$ activity, resulting in a significant reduction in progesterone output by the corpora lutea $(\mathrm{CL})$ as no adverse effects were seen with respect to the maturity rate of oocytes, fertilization rates, embryo quality, and reproductive outcomes during the subsequent replacement of frozen embryos derived from women who had received a GnRHa trigger (3-7). Following these first disappointing results several studies have shown a LP rescue with the use of a modified LPS, resulting in reproductive outcomes comparable to those observed after hCG triggering $(3,4,8-10)$. Up until now, the method of modified LPS reported by Humaidan et al. (3) appears to be the most frequently used regimen in fresh embryo transfer cycles (11). However, further fine-tuning of LPS after the GnRHa trigger could still possibly be warranted (12-14). Significantly increased implantation rates (IRs) were previously reported in oocyte recipients as well as in patients who were triggered with hCG, if they received a single mid-luteal bolus of GnRHa in addition to standard LPS (15-17). However, others have failed to support this finding $(18,19)$. Importantly, there are significant differences in the early-mid-LP endocrine pattern when GnRHa triggers and hCG triggers are compared, especially in terms of LH levels. From this, it could be hypothesized that the GnRHa-triggered IVF cycle could benefit more from the addition of a bolus of GnRHa to boost the circulating endogenous LH and thus, progesterone levels around the time of implantation than the hCG triggered cycle. No studies previously investigated this issue. Therefore, the aim here was to explore a possible fine-tuning of the LPS of GnRHa-triggered IVF/ICSI cycles, using the previously suggested protocol of Humaidan et al. (3). Here, we have combined a GnRHa trigger and the 1,500 IU HCG dose administered on the day of oocyte pick-up (OPU) $(3,4)$ with a single dose of GnRHa administered 6 days after OPU, i.e., 2 days before the expected day of implantation in an attempt to mimic the natural cycle peak of progesterone during the midLP. In addition, a standard LPS consisting of vaginal progesterone (P) and oral estrogen (E2) was used. By possibly optimizing the early and mid-LP in terms of steroids, it was also hypothesized that we would subsequently optimize IRs, which was the primary outcome measure of the study.

\section{MATERIALS AND METHODS}

\section{Study Design}

This single-center, prospective, randomized, controlled trial was conducted from February 2014 to January 2016 at the IVF center Ibn Rochd, Constantine, Algeria. All patients provided written informed consent to participate in the study, which was conducted in accordance with the Declaration of Helsinki and Good Clinical Practice. The research project was approved by the Ethics Committee of the University Hospital Centre Ibn Badis, Constantine. Moreover, the study was registered in ClinTrial.gov, Number: 02053779.

\section{Study Participants}

A total of 328 women were included in the study. Women were enrolled into the study if they fulfilled the following criteria: aged $<40$ years; baseline FSH $<12$ IU/L; no uterine fibroids, Mullerian malformations, ovarian (endometrioma), or adnexal (hydrosalpinx) abnormalities. In addition, patients needed to have at least one embryo for fresh transfer. The exclusion criteria were a very high risk of ovarian hyper stimulation syndrome (OHSS) development ( $>30$ follicles $>11 \mathrm{~mm}$ on the day ovulation was triggered), poor responders identified according to Bologna criteria, or having a partner with azoospermia.

\section{Randomization}

Women meeting the inclusion criteria were randomized per block of four patients according to a computer-generated randomization list. The randomization was performed by a nurse on the day of embryo transfer (ET) 2-3 days after OPU. The researchers were blinded to group allocations. Patients were assigned to one of two LPS groups. The study group received a single-dose of GnRHa (triptorelin $0.1 \mathrm{mg}$ s.c.) on day 6 after OPU administered by either a nurse or by the patient herself, in addition to standard LPS; the control group received standard LPS, only.

\section{Hormonal Treatment and Ovulation Trigger}

The ovarian stimulation was initiated from day 3 of the menstrual cycle and continued until the day that ovulation was induced. The standard daily starting dose of recombinant human FSH (Gonal-Fं; Merck Serono) or (Puregon ${ }^{\circledR}$; MSD) was 150-225 IU, depending on patient age, BMI, antral follicle count, and basal serum FSH levels. After 5 days, doses were adjusted according to ovarian response. Once the leading follicle had reached a size of $13 \mathrm{~mm}$, co-treatment with a GnRH antagonist (Cetrotide ${ }^{\circledR}$ $0.25 \mathrm{mg}$; Merck Serono) or (Orgalutran ${ }^{\circledR} 0.25 \mathrm{mg}$; MSD) was initiated and continued up until and including the day of induction of ovulation. When at least three follicles had reached a size of $17 \mathrm{~mm}$, ovulation induction was performed with a single bolus of $0.2 \mathrm{mg}$ triptorelin, s.c. (Decapeptyl ${ }^{\circledR} 0.1 \mathrm{mg}$, Ipsen, France), followed by OPU $36 \mathrm{~h}$ later. Retrieved oocytes were fertilized by either IVF or ICSI, depending on sperm quality.

\section{Embryo Transfer and LPS}

According to the local regional policy and after an agreement between the patient and the medical team, one to three embryos were transferred.

For LPS, in addition to a bolus of hCG 1,500 IU, IM (Pregnyl ${ }^{\circledR}$; MSD) given $1 \mathrm{~h}$ after OPU, all patients received micronized $\mathrm{P}$ $\left(600 \mathrm{mg} /\right.$ day) vaginally (Utrogestan ${ }^{\circledR}$; Laboratoires BesinsIscovesco, Paris, France) and estradiol (4 mg/day) orally (Progynova 2 mg; Schering, Madrid, Spain), beginning on the day after oocyte retrieval and continuing until either a fetal 
heartbeat was detected on ultrasound at 5 weeks after OPU or a negative pregnancy test.

\section{Blood Samples and Hormone Assays}

Blood sampling was performed for FSH, LH, TSH, E2, and prolactin on day 1 of stimulation, for $\mathrm{E} 2, \mathrm{P}$, and $\mathrm{LH}$ on the day of ovulation induction, for E2, P, LH, and FSH on OPU day +7 , within 14-16 $\mathrm{h}$ following the Triptorelin $0.1 \mathrm{mg}$ injection particularly in the study group. Beta hCG was measured 14 days after OPU. Sera were analyzed immediately using a Vidas kit (BioMerieux, France). All measurements were performed according to the manufacturer's instructions.

\section{Outcomes and Measures}

The primary endpoint determining efficacy was IR. Secondary endpoints were the mid-luteal steroid levels, the rate of early pregnancy loss, the rate of clinical pregnancy, the rate of ongoing pregnancy $(\mathrm{OP})$, the live birth (LB) rate, and the incidence of OHSS. The IR was defined as the number of gestational sacs with a fetal heartbeat, as assessed by ultrasound at 5 weeks after OPU, divided by the number of embryos transferred. Clinical pregnancy was defined as a positive serum $\beta$-hCG test with ultrasound evidence of a gestational sac and fetal heart beat at 5 weeks after OPU. OP was defined as pregnancy progressing beyond 12 weeks after OPU.

A LB was defined as a delivery of a live baby beyond 26 weeks after OPU. Early pregnancy loss was defined as a positive serum $\beta$-hCG test without ultrasound evidence of a gestational sac or a gestational sac without a fetal heartbeat.

\section{Sample Size and Power Calculation}

Based on previously published data (15), which shows that the administration of a bolus of GnRHa 6 days after ICSI in oocyte recipients increases the IR from 25.1 to $36.9 \%$, it was hypothesized that a similar increase might be expected in patients undergoing ovarian stimulation. Using these findings, the sample size was calculated using a two-sided significance level of 0.05 and a power of $80 \%$ to detect a minimum difference of $10 \%$ for the endpoint (IR) between the study group and the control group. By assuming a mean of two embryos transferred per patient, a total of 328 embryos (164 patients) were needed in each group.

\section{Statistical Methods}

Chi-squared or Fisher's exact tests were used for categorical variables where appropriate. An independent sample $t$-test was used for continuous variables that were normally distributed, and the Mann-Whitney $U$-test was used for data not normally distributed. Data are presented as means \pm SD unless otherwise stated. Multivariate logistic regression analyses to determine the effect of the GnRHa on the OP and LB was performed in the GnRHa arm. Potential predictive factors were identified by univariate logistic regression per candidate predictive factor using a $P$ value of $<0.2$ as a criterion for exclusion. Candidate predictive factors that were evaluated including age, basal $\mathrm{LH}$, prolactin on day 2 of the cycle, duration of stimulation, total dose of r-FSH, total dose of antagonist, ovarian response to stimulation, E2 and LH levels on the day of triggering, embryo quality, number of transferred embryos, mid luteal LH level, and the number of vitrified embryos. The ovarian response was dichotomized into two groups, in order to simplify the interpretation and application of the model, according to the number of follicles $\geq 11 \mathrm{~mm}$ observed on the day of trigger (20): normal responders (NR; $\leq 13$ follicles) and high responders (HR; 14-30 follicles). Out of all 328 patients, 178 were NR and 150 were HR. In the GnRHa arm, 100 patients were NR and 65 were HR. All the above covariates were examined for independence. The model was performed with the backward procedure. For both outcomes (OP and LB), the model is presented with the odds ratio (OR), 95\% confidence interval (CI), and $P$ value for each predictor. The area under the curve (AUC) was calculated to assess the model's discriminative capacity. All $P$-values quoted are two-sided, and $P<0.05$ was considered statistically significant. The SPSS statistical package (Release 24.0; SPSS, Inc.) was used for statistical evaluation.

\section{RESULTS}

The trial was performed according to CONSORT guidelines. The flow of participants is shown in Figure 1. A total of 341 patients met the study criteria and were recruited. However, 13 patients were subsequently excluded, 6 patients due to hyper-responsiveness to stimulation ( $>30$ follicles), 4 patients due to fertilization failure or failed embryo development, and 3 patients for other reasons. Therefore, a total of 328 patients were randomized into the study group $(n=165)$ or the control group $(n=163)$ on the day of ET. None of these patients were lost at follow-up; therefore, a total of 165 patients in the study group and 163 patients in the control group were included in the main analyses.

\section{Demographic Data and Cycle Characteristics}

The baseline characteristics of the patients and the outcomes of ovarian stimulation are shown in (Table 1). No significant differences were seen between the study and the control group. Fertilization rates and the number and quality of embryos transferred were also similar in both groups (Table 1).

\section{Serum Hormone Levels}

Endocrine profiles are depicted in Table 2. Serum levels of FSH, $\mathrm{LH}$, and E2 at the start (day 1 of stimulation) and at the end of ovarian stimulation were similar in treatment and control groups. However, serum concentrations of FSH, LH, P, and E2 during the LP on OPU +7 were significantly higher in the GnRHasupplemented group compared to the control group.

\section{Reproductive Outcomes}

No significant differences were observed in IRs [27 vs. $23 \%$ OR 1.2 (95\% CI 0.9-1.7)], percentage of positive pregnancy tests [46 vs. $42 \%$ OR 1.2 (95\% CI 0.7-1.8)], clinical pregnancy rate [38 vs. $31 \%$ OR 1.3 (95\% CI $0.8-2.1)$ ], OP rate [37 vs. $31 \%$ OR $1.3(95 \%$ CI $0.8-2.1$ )], LB rate [36 vs. $31 \%$ OR 1.3 (95\% CI $0.8-2.0)$ ], and early pregnancy loss rate [ 16 vs. $27 \%$ OR 0.5 (95\% CI $0.2-1.2)$ ], in the study and control group, respectively (Table 3 ). The 


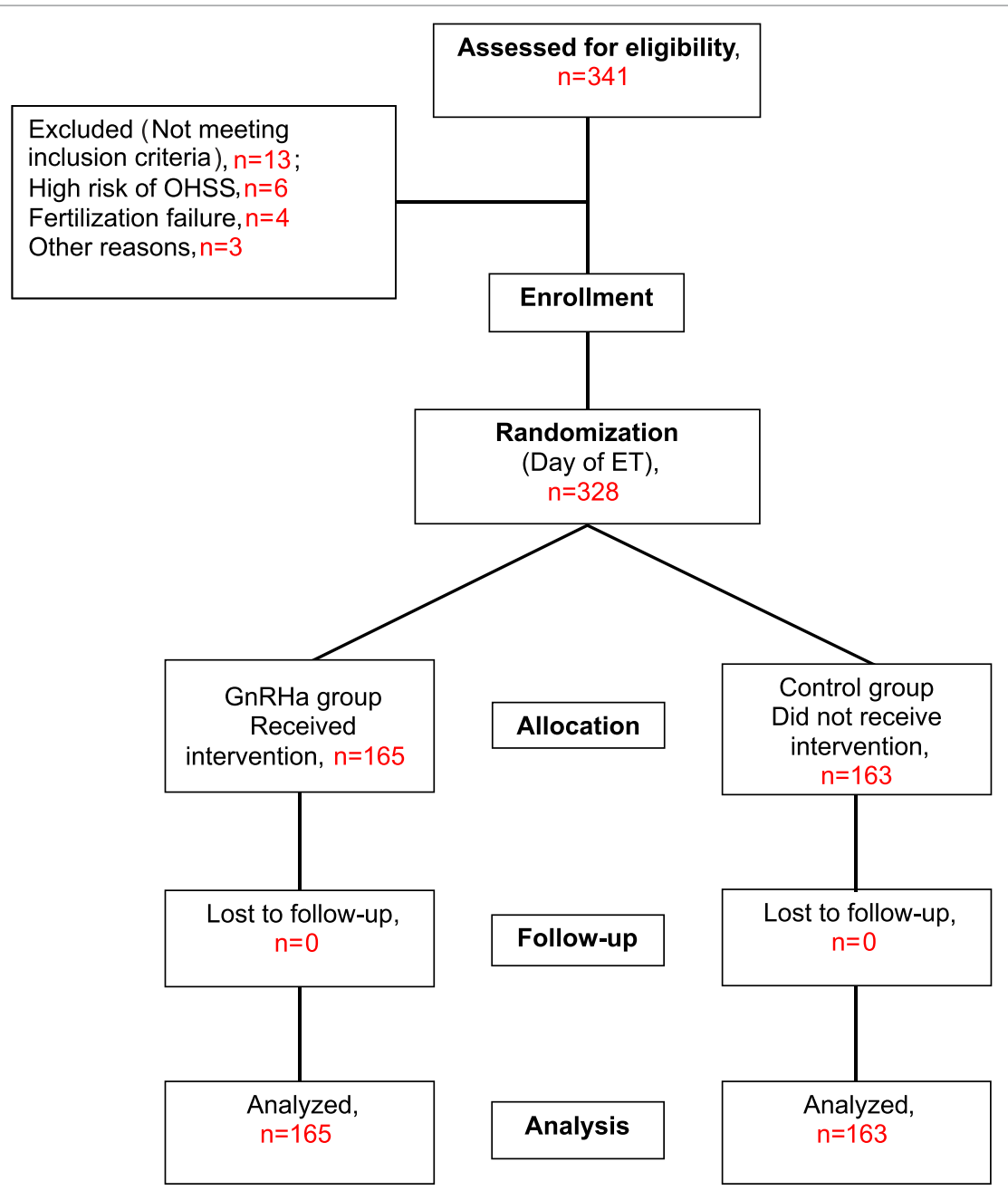

FIGURE 1 | Study flow chart. Benmachiche. Mid-luteal GnRHa in GnRHa triggered cycles.

TABLE 1 | Baseline characteristics and stimulation outcome.

\begin{tabular}{lccc}
\hline Variable & $\begin{array}{c}\text { GnRHa } \\
\text { (OPU +6) }\end{array}$ & Control & $\begin{array}{c}\boldsymbol{P} \\
\text { value }\end{array}$ \\
\hline Patients, $n$ & 165 & 163 & \\
Age (years) & $31.7 \pm 3.6$ & $32.1 \pm 4.1$ & NS \\
Body mass index (kg/m ${ }^{2}$ ) & $27.8 \pm 4.8$ & $28.2 \pm 4.5$ & NS \\
Duration of follicle-stimulating & $9.1 \pm 1.2$ & $9.3 \pm 1.1$ & NS \\
hormone (FSH) stimulation (days) & & & \\
Total dose of FSH (IU) & $1,831.1 \pm 317.0$ & $1,844.3 \pm 294.7$ & NS \\
Total dose of antagonist (mg) & $0.9 \pm 0.2$ & $1.0 \pm 0.2$ & NS \\
Endometrium (mm) & $10.7 \pm 1.4$ & $10.3 \pm 1.5$ & NS \\
Follicles the day of triggering, $n$ & $12.2 \pm 5.8$ & $13.5 \pm 6.4$ & NS \\
Oocytes, $n$ & $9.0 \pm 4.5$ & $9.9 \pm 5.4$ & NS \\
Mll oocytes, $n$ & $7.2 \pm 3.8$ & $8.1 \pm 4.8$ & NS \\
2PN zygotes, $n$ & $5.4 \pm 2.8$ & $6.1 \pm 3.7$ & NS \\
Embryos, $n$ & $5.1 \pm 2.8$ & $5.7 \pm 3.4$ & NS \\
Transferred embryos, $n$ & $2.3 \pm 0.5$ & $2.4 \pm 0.8$ & NS \\
Vitrified embryos, $n$ & $0.7 \pm 1.6$ & $0.9 \pm 1.8$ & NS \\
\hline Values are mean $n$ SD $n$ & & & \\
\hline
\end{tabular}

Values are means $\pm S D$. NS $=$ Not significant.

GnRHa, gonadotropin-releasing hormone agonist; MII, metaphase II; PN, pro-nuclei. results of the Multivariate logistic regression analysis are given in (Table 4). In the luteal GnRHa arm, 62/165 (37.6\%) patients had an OP and 60/165 (36.4\%) had a LB. By studying, the coefficients related to each explanatory variable individually, at a risk of $5 \%$ we found that five factors increased the likelihood of OP: normo-responders (NR), higher pre-ovulatory LH levels, higher mid-luteal LH levels, good embryo quality, and a higher number of embryos transferred. The same factors, except for the number of transferred embryos, had a predictive value on the LB. The areas under the receiver operating characteristic (ROC) curve for $\mathrm{OP}$ and $\mathrm{LB}$ were 0.80 and 0.70 , respectively.

\section{Ovarian Hyper Stimulation Syndrome}

No early onset OHSS cases were seen in the two groups. No late onset OHSS cases were seen in NR patients ( $\leq 13$ follicles). However, two moderate-late-onset OHSS cases occurred in the HR subgroup (14-30 follicles). One case occurred in the luteal GnRHa group, with the other in the control group. Both patients were shortly hospitalized (24-48 h) with only symptomatic relief. 


\section{DISCUSSION}

This randomized controlled study explored the possible effect of a mid-luteal GnRHa bolus on IRs in IVF/ICSI patients. Overall, no significant differences were seen in implantation and clinical pregnancy rates. In contrast, significantly higher mid-luteal steroid levels were seen in the group of patients who received a mid-luteal bolus of GnRHa (Table 2). Two cases of moderate-late onset OHSS occurred; one case in the study group and one in the control group. Both of these cases were associated with twin pregnancies (Table 3).

Several studies have previously shown a positive effect of GnRHa administration during the LP. The evidence for its efficacy is, however, of low quality due to the heterogeneity between trials (21-24). In the present study, the IR, OP, and LB rates were slightly higher in the GnRHa group compared to the control group [27 vs. $23 \%$ OR 1.2 (95\% CI $0.9-1.7), P<0.27$ ], [ 37 vs. $31 \%$ OR 1.3 (95\% CI $0.8-2.1), P<0.23$ ], and [ 36 vs. $31 \%$ OR 1.3 (95\% CI $0.8-2.0$ ), $P<0.27$ ], respectively; however, the differences fell short of reaching statistical significance (Table 3). However, an absolute risk difference of $5 \%$ in LB rate - although not significant - is clinically relevant. In fact, if sample size had been calculated taking OP as the main outcome, which could be regarded more relevant in IVF cycles than IR, we would have needed twice the number of patients

TABLE 2 | Endocrine parameters.

\begin{tabular}{|c|c|c|c|}
\hline Variable & GnRHa (OPU + 6) & Control & $P$ value \\
\hline Patients, $n$ & 165 & 163 & \\
\hline Basal FSH (IU/L) & $5.9 \pm 1.7$ & $5.8 \pm 1.7$ & 0.32 \\
\hline Basal LH (IU/L) & $5.1 \pm 3.3$ & $5.2 \pm 3.1$ & 0.67 \\
\hline Basal E2 (pg/mL) & $42.0 \pm 20.9$ & $58.8 \pm 198.0$ & 0.28 \\
\hline Basal Prolactin (ng/mL) & $21.5 \pm 19.4$ & $20.9 \pm 12.7$ & 0.74 \\
\hline LH (IU/L) day of triggering & $1.3 \pm 1.0$ & $1.2 \pm 0.8$ & 0.40 \\
\hline $\mathrm{P}(\mathrm{ng} / \mathrm{mL})$ day of triggering & $1.1 \pm 0.6$ & $1.1 \pm 0.6$ & 0.92 \\
\hline E2 $(\mathrm{pg} / \mathrm{mL})$ day of triggering & $1,852.7 \pm 889.7$ & $2,034.8 \pm 917.6$ & 0.07 \\
\hline $\mathrm{FSH} O P U+7(\mathrm{IU} / \mathrm{L})^{\mathrm{a}}$ & $0.9 \pm 0.4$ & $0.6 \pm 0.3$ & 0.001 \\
\hline $\mathrm{LH} O P U+7(\mathrm{IU} / \mathrm{L})^{\mathrm{a}}$ & $4.1 \pm 2.1$ & $1.4 \pm 1.4$ & 0.001 \\
\hline $\mathrm{E} 2 \mathrm{OPU}+7(\mathrm{pg} / \mathrm{mL})^{\mathrm{a}}$ & $1,274.3 \pm 861.1$ & $971.4 \pm 849.6$ & 0.001 \\
\hline $\mathrm{P}$ OPU $+7(\mathrm{ng} / \mathrm{mL})^{\mathrm{a}}$ & $53.3 \pm 33.5$ & $37.6 \pm 23.0$ & 0.001 \\
\hline$\beta$-hCG OPU +14 (mIU/mL) & $245.3 \pm 377.0$ & $191.7 \pm 342.1$ & 0.17 \\
\hline
\end{tabular}

Values are mean $\pm S D$, unless otherwise noted.

aStatistically significant difference between groups, with $P<0.05$.

OPU, oocyte pick-up; FSH, follicle-stimulating hormone; $L H$, luteinizing hormone; $P$,

progesterone; E2, estradiol; hCG, human chorionic gonadotropin. included in this study and results might have been statistically significant in favor of the intervention group (at least this is the trend).

In 2004, Tesarik et al. demonstrated that a single supplementary bolus of a GnRHa as LPS in oocyte recipient cycles without pituitary desensitization-improved the IR (36.9 vs. $25.1 \%)$ and LB (31.1 vs. $21.5 \%)$. In 2006, the same investigators evaluated the effects of $0.1 \mathrm{mg}$ triptorelin administration 6 days after ICSI in GnRHa $(n=300)$ and GnRH antagonist $(n=300)$ cycles. IRs were significantly increased with both regimens. However, only in GnRH antagonist cycles a significant increase in the OP was observed (16). Subsequent studies demonstrated that LP GnRHa supplementation seemed to benefit the GnRH antagonist cotreated cycle $(8,16,25-27)$ more than the long GnRHa co-treated cycle $(18,19,28)$. The main reason for this is the down-regulation of the pituitary, which is induced by the long GnRHa protocol (28). In support of this conclusion, Kung et al. (29) recently verified that luteal $\mathrm{GnRHa}$ administration significantly increased clinical pregnancy and LB rates in $\mathrm{GnRH}$ antagonist co-treated patients, but not in patients undergoing a long GnRHa downregulation protocol. Interestingly, Kung et al. (29) suggested that patients with higher basal FSH levels $(>8 \mathrm{mIU} / \mathrm{mL})$ and reduced numbers of mature oocytes $(\leq 3)$ might have better outcomes when receiving LP support with GnRHa.

In the present study, although we demonstrated a significant increase in mid-luteal endogenous gonadotropins and steroids in the study group, we failed to reject the null hypothesis in terms of implantation and pregnancy rates. A subsequent question remaining to be answered is if there were any confounding factors impacting the clinical effects of the intervention. To explore this issue, we conducted a multivariate regression analysis, exploring variables that could independently correlate with the occurrence of OP and LB in the luteal GnRHa arm. Implantation was not chosen as an outcome parameter of the model, as the transfer of more than one embryo could partially compensate for early pregnancy losses, moreover, the prediction of OP and LB was more clinically relevant. In the study population (GnRHa group), $37.6 \%(62 / 165)$ of women had an OP and $36.4 \%(60 / 165)$ had a LB. The results of the multivariate regression (Table 4) show that the ovarian response might confound the relationship between the mid-luteal GnRHa dose and the pregnancy outcome. The NR patient was three times more likely to have OP $[P<0.005$, OR 3.18 (95\% CI 1.41-7.17)] and LB $[P<0.002$, OR 3.37 (95\% CI $1.56-7.27)]$, respectively, compared to the HR patient. Indeed, the

TABLE 3 | Overall reproductive outcome.

\begin{tabular}{|c|c|c|c|c|}
\hline Variable & GnRHa (OPU + 6) & Control & OR (95\% Cl) & $P$ value \\
\hline Patients, $n$ & 165 & 163 & & \\
\hline Positive pregnancy, $n$ (\%) & $76 / 165(46)$ & $69 / 163(42)$ & $1.2(0.7-1.8)$ & 0.49 \\
\hline Implantation rate, $n(\%)$ & $104 / 381(27)$ & 92/386 (23) & $1.2(0.9-1.7)$ & 0.27 \\
\hline Clinical pregnancy rate, $n(\%)$ & 63/165 (38) & $51 / 163(31)$ & $1.3(0.8-2.1)$ & 0.19 \\
\hline Ongoing pregnancy rate, $n(\%)$ & $62 / 165(37)$ & $51 / 163(31)$ & $1.3(0.8-2.0)$ & 0.23 \\
\hline Delivery rate, $n(\%)$ & $60 / 165(36)$ & $50 / 163(31)$ & $1.3(0.8-2.0)$ & 0.27 \\
\hline Early miscarriage rate, $n$ (\% of positive hCG) & $13 / 76(17)$ & $19 / 69(27)$ & $0.5(0.2-1.2)$ & 0.13 \\
\hline $\mathrm{OHSS}^{\mathrm{a}}, n(\%)$ & $1 / 165(0.6)$ & $1 / 163(0.6)$ & $1.0(0.1-16.0)$ & 0.99 \\
\hline
\end{tabular}

Values are number (percentage). There were no significant differences between the two groups.

${ }^{a}$ Moderate late ovarian hyper stimulation syndrome (OHSS). 
TABLE 4 | Multivariate regression results: the effect of mid luteal GnRHa on ongoing pregnancy (OP) and live birth (LB): patients in the GnRHa arm.

\begin{tabular}{|c|c|c|c|c|c|c|}
\hline \multirow[t]{2}{*}{ Variable } & \multirow[t]{2}{*}{ OR } & $(95 \% \mathrm{Cl})$ & \multirow[t]{2}{*}{$P$ Value } & \multirow[t]{2}{*}{ OR adj. } & $(95 \% \mathrm{Cl})$ & \multirow[t]{2}{*}{$P$ value } \\
\hline & & Univariate analysis & & & Multivariate analysis & \\
\hline \multicolumn{7}{|l|}{ OPa $^{a}$} \\
\hline Basal LH (mlU/mL) & 0.90 & $0.80-1.02$ & 0.11 & 0.86 & $0.74-1.01$ & 0.08 \\
\hline Total dose of r-FSH (IU) & 1.00 & $1.000-1.002$ & 0.07 & 1.001 & $1.000-1.002$ & 0.06 \\
\hline Ovarian response status (NR vs. HR) & 2.61 & $1.31-5.20$ & 0.006 & 3.18 & $1.41-7.17$ & 0.005 \\
\hline $\mathrm{LH}(\mathrm{mlU} / \mathrm{mL})$ day of triggering & 1.34 & $0.98-1.83$ & 0.06 & 1.75 & $1.16-2.63$ & 0.007 \\
\hline $\mathrm{LH}(\mathrm{mlU} / \mathrm{mL})$ day OPU +7 & 1.15 & $0.89-1.33$ & 0.08 & 1.25 & $1.04-1.50$ & 0.01 \\
\hline Embryo quality (Good vs. Bad) & 2.40 & $0.91-6.30$ & 0.08 & 3.67 & $1.22-11.06$ & 0.02 \\
\hline Transferred embryos, $n$ & 1.63 & $0.92-2.91$ & 0.09 & 2.11 & $1.07-4.19$ & 0.03 \\
\hline \multicolumn{7}{|l|}{ LB $^{b}$} \\
\hline Ovarian response status (NR vs. HR) & 2.41 & $1.21-4.80$ & 0.01 & 3.37 & $1.56-7.27$ & 0.002 \\
\hline $\mathrm{LH}(\mathrm{mlU} / \mathrm{mL})$ day of triggering & 1.34 & $0.98-1.83$ & 0.06 & 1.65 & $1.12-2.43$ & 0.01 \\
\hline $\mathrm{LH}(\mathrm{mlU} / \mathrm{mL})$ day OPU + 7 & 1.13 & $0.97-1.32$ & 0.11 & 1.65 & $1.12-2.43$ & 0.01 \\
\hline Embryo quality (Good vs. Bad) & 2.25 & $0.85-5.93$ & 0.10 & 3.53 & $1.24-10.05$ & 0.01 \\
\hline
\end{tabular}

The receiver operating characteristic curve (AUC) for OP: 0.80 .

${ }^{b}$ The receiver operating characteristic curve (AUC) for $L B: 0.70$.

OR, odds ratio; OR adj, adjusted odds ratio; $\mathrm{Cl}$, confidence interval 95\%; NR, normo responders; HR, high responders.

OP rate increased from $9.70 \%(16 / 165)$ in HR to $27.88 \%(46 / 165)$ in NR and the LB rate increased from $9.70 \%(16 / 165)$ in HR to $26.66 \%(44 / 165)$ in NR. The reason for this discrepancy may be attributed to a possible threshold of mid-luteal steroids compatible with optimal endometrial receptivity (30-35). Moreover, the availability of good embryo quality, a pre-ovulatory, and mid-luteal LH activities were also associated with increased odds for both OP and LB. Finally, as expected, the number of embryos transferred was independently associated with the occurrence of OP (Table 4). The areas under the ROC curve for OP and LB were 0.80 and 0.70 , respectively, indicating that both models fit moderately well and are clinically useful, and as results provide a tool with which to inform patients of the prognosis after the mid luteal addition of the GnRHa. Thus, the addition of a mid-luteal bolus GnRHa to NR (<14 follicles) but not to HR (14-30) seems to be the fine-tuning sought to optimize the protocol suggested by Humaidan et al. $(3,4)$ in terms of reproductive outcome in both categories. Whereas, in the very $\mathrm{HR}$ (>30 follicles) category, which was excluded from the current study, the freeze all strategy remains best option in our opinion.

The exact mechanism behind the presumed beneficial effect of LP GnRHa administration remains poorly defined. It has been hypothesized that GnRHa either supports CL function by inducing LH secretion by the pituitary gonadotrophic cells $(8,26)$ or stimulates the endometrial GnRH receptors (36). Tesarik et al. (15) postulated a direct effect of GnRHa on the embryo, as suggested by an increase in $\beta$-HCG secretion. The latter mechanism does not seem to be supported by our study because there were no significant differences in hCG levels between study and control groups (Table 2). Interestingly, the findings of the present study show that a bolus of GnRHa, when administered 6 days after OPU in GnRH antagonist cycles, is able to induce a surge of pituitary gonadotropins (FSH and $\mathrm{LH}$ ), eliciting an increase in steroid production (E2 and $\mathrm{P}$ ) by the CL (Table 2 ). The above-mentioned hormone variations were still detectable within $24 \mathrm{~h}$ following the GnRHa administration; $14-16 \mathrm{~h}$ in our protocol precisely. Thus, the increase of FSH, LH, P, and E2 was significantly higher in the study group compared to the control group (Table 2), which is in agreement with previous reports $(8,26)$. However, similar changes in the hormonal luteal profile have not been reported in other studies $(16,37)$.

In terms of safety, we did not observe any increase in the development of OHSS in GnRHa-treated patients, which is in agreement with Tesarik et al. (16). Thus, this regimen could be considered a safe approach to support the LP with LH activity when comparing with mid-luteal addition of low dose of hCG (38).

The limitations of the present study are, firstly, from a clinical viewpoint, $\mathrm{OP}$ and LB are more relevant outcomes in IVF cycles than IR $(39,40)$. IR will equate with clinical pregnancy rate only when single embryo transfer (SET) is used (41). In the present study, only 5\% (19/328) of cycles were SET cycles. If a clinical pregnancy was considered as the main outcome, the number of patients required would be twice that of the present study (more than 600), which would be beyond the capacity of a single fertility center. Secondly, as the secondary data analyzed used small numbers reported from a single center, this limited generalizability and so these data should be interpreted with caution and be considered only as preliminary to a larger future study.

In conclusion, the present study demonstrates that a single dose of GnRHa administered 6 days after OPU in GnRHa-triggered IVF cycles supplemented with a small bolus of hCG on the day of OPU did not improve reproductive outcomes in the study group as compared to controls, despite the fact that significantly higher mid-luteal endogenous gonadotropins and steroid levels were present in the study group. Results of the multivariate regression analysis performed in the luteal GnRHa population support the concept of "individualized" ovarian response-based luteal support, suggesting that the above-mentioned regimen appears to be a promising alternative to provide an optimal level of LH activity throughout the early-mid-LP to maximize each patient's chance of a pregnancy with minimal safety issues. The findings need further corroboration in a large-scale study. 


\section{ETHICS STATEMENT}

All patients provided written informed consent to participate in the study, which was conducted in accordance with the Declaration of Helsinki and Good Clinical Practice. The research project was approved by the Ethics Committee of the University hospital Centre Ibn Badis, Constantine. Moreover, the study was registered in ClinTrial.gov, Number: 02053779.

\section{AUTHOR CONTRIBUTIONS}

$\mathrm{ABenmachiche}$ and $\mathrm{PH}$ designed the study, drafted, and edited the manuscript. ABenmachiche performed data collection, handling of data, and statistical analysis. AZ, SB, and ABoularak

\section{REFERENCES}

1. Humaidan P, Bredkjaer HE, Bungum L, Bungum M, Grøndahl ML, Westergaard L, et al. GnRH agonist(buserelin) or hCG for ovulation induction inGnRHantagonistIVF/ICSI cycles: a prospective randomized study. Hum Reprod (2005) 20(5):1213-20. doi:10.1093/humrep/deh765

2. Kolibianakis EM, Schultze-Mosgau A, Schroer A, et al. A lower ongoing pregnancy rate can be expected when GnRH agonist is used for triggering final oocyte maturation instead of HCG in patients undergoing IVF with GnRH antagonists. Hum Reprod (2005) 20(10):2887-92. doi:10.1093/humrep/ dei150

3. Humaidan P, Bungum L, Bungum M, Yding AC. Rescue of corpus luteum function with peri-ovulatory HCG supplementation in IVF/ICSI GnRH antagonist cycles in which ovulation was triggered with a GnRH agonist: a pilot study. Reprod Biomed Online (2006) 13:173-8. doi:10.1016/S1472-6483(10)60612-8

4. Humaidan P, Ejdrup BH, Westergaard LG, Yding AC. 1,500 IU human chorionic gonadotropin administered at oocyte retrieval rescues the luteal phase when gonadotropin-releasing hormone agonist is used for ovulation induction: a prospective, randomized, controlled study. Fertil Steril (2010) 93:847-54. doi:10.1016/j.fertnstert.2008.12.042

5. Humaidan P, Kol S, Papanikolaou EG. GnRH agonist for triggering of final oocyte maturation: time for a change of practice? Hum Reprod Update (2011) 17:510-24. doi:10.1093/humupd/dmr008

6. Humaidan P, Papanikolaou EG, Kyrou D, Alsbjerg B, Polyzos NP, Devroey P, et al. The luteal phase after GnRH-agonist triggering of ovulation: present and future perspectives. Reprod Biomed Online (2012) 24:134-41. doi:10.1016/j. rbmo.2011.11.001

7. Humaidan P. Luteal phase rescue in high-risk OHSS patients by GnRHa triggering in combination with low-dose HCG: a pilot study. Reprod Biomed Online (2009) 18(5):630-4. doi:10.1016/S1472-6483(10)60006-5

8. Pirard C, Donnez J, Loumaye E. GnRH agonist as luteal phase support in assisted reproduction technique cycles: results of a pilot study. Hum Reprod (2006) 21(7):1894-900. doi:10.1093/humrep/del072

9. Engmann L, DiLuigi A, Schmidt D, Nulsen J, Maier D, Benadiva C. The use of gonadotropin-releasing hormone $(\mathrm{GnRH})$ agonist to induce oocyte maturation after cotreatment with GnRH antagonist in high-risk patients undergoing in vitro fertilization prevents the risk of ovarian hyperstimulation syndrome: a prospective randomized controlled study. Fertil Steril (2008) 89:84-91. doi:10.1016/j.fertnstert.2007.02.002

10. Papanikolaou EG, Verpoest W, Fatemi H, Tarlatzis B, Devroey P, Tournaye H. A novel method of luteal supplementation with recombinant LH, when a GnRH-Agonist is used instead of HCG for ovulation triggering. A randomized prospective proof of concept study. Fertil Steril (2011) 3:1174-7. doi:10.1016/j. fertnstert.2010.09.023

11. de Ziegler D, Shoham Z. Survey results: triggering of ovulation with GnRh-a in ART: worldwide feedback on an emerging new option with great potential. IVF Worldwide Newsletter. (2013). Available from: www.ivf-worldwide.com

12. Garcia-Velasco JA, Motta L, López A, Mayoral M, Cerrillo M, Pacheco A. Low-dose human chorionic gonadotropin versus estradiol/progesterone actively participated in data acquisition, handling of data and manuscript drafting. All co-authors accepted the final draft.

\section{ACKNOWLEDGMENTS}

The authors thank Abdelhamid Aberkane as a president of a Medical Research Ethics Committee (University Hospital Benbadis, Constantine) for his valuable assistance. Statistical analyses were performed by Abdesslem Brihmat.

\section{FUNDING}

This study was supported by the center for Reproductive Medicine Ibn-Rochd.

luteal phase support in gonadotropin-releasinghormone agonist-triggered assistedreproductive technique cycles: understanding a new approach. Fertil Steril (2010) 94(7):2020-3. doi:10.1016/j.fertnstert.2010.06.035

13. Humaidan P, Alsbjerg B. GnRHa trigger for final oocyte maturation: is HCG trigger history? Reprod Biomed Online (2014) 29:274-80. doi:10.1016/j. rbmo.2014.05.008

14. Humaidan P, Polyzos NP. Human chorionic gonadotropin vs. Gonadotropinreleasing hormone agonist trigger in assisted reproductive technology"The king is dead, long live the king!". Fertil Steril (2014) 102(2):339-41. doi:10.1016/j.fertnstert.2014.04.047

15. Tesarik J, Hazout A, Mendoza C. Enhancement of embryo developmental potential by a single administration of $\mathrm{GnRH}$ agonist at the time of implantation. Hum Reprod (2004) 19(5):1176-80. doi:10.1093/humrep/deh235

16. Tesarik J, Hazout A, Mendoza-Tesarik R, Mendoza N, Mendoza C. Beneficial effect of luteal-phase GnRH agonist administration on embryo implantation after ICSI in both GnRH agonist- and antagonist-treated ovarian stimulation cycles. Hum Reprod (2006) 21(10):2572-9. doi:10.1093/humrep/del173

17. Razieh DF, Maryam AR, Nasim T. Beneficial effect of luteal-phase gonadotropin-releasing hormone agonist administration on implantation rate after intracytoplasmic sperm injection. Taiwan JObstet Gynecol (2009) 48(3):245-8. doi:10.1016/S1028-4559(09)60297-7

18. Ata B, Yakin K, Balaban B, Urman B. GnRH agonist protocol administration in the luteal phase in ICSI-ET cycles stimulated with the long GnRH agonis protocol: a randomized, controlled double blind study. Hum Reprod (2008) 23(3):668-73. doi:10.1093/humrep/dem421

19. Inamdar DB, Majumdar A. Evaluation of the impact of gonadotropin-releasing hormone agonist as an adjuvant in lutealphasesupport on IVF outcome. J Hum Reprod Sci (2012) 5:279-84. doi:10.4103/0974-1208.106341

20. Papanikoloaou EG, Tournaye H, Verpoest W, Camus M, Vernaeve V, Steirteghem AV, et al. Early and late ovarian hyperstimulation syndrome: early pregnancy outcome and profile. Hum Reprod (2005) 20(3):636-41. doi:10.1093/humrep/deh638

21. Oliveira JB, Baruffi R, Petersen CG, Mauri AL, Cavagna M, Franco JG Jr. Administration of single-dose GnRH agonist in the luteal phase in ICSI cycles: a meta-analysis. Reprod Biol Endocrinol (2010) 8:107. doi:10.1186/ 1477-7827-8-107

22. Kyrou D, Kolibianakis EM, Fatemi HM, Tarlatzi TB, Devroey P, Tarlatzis BC. Increased live birth rates with GnRH agonist addition for luteal support in ICSI/IVF cycles: a systematic review and meta-analysis. Hum Reprod Update (2011) 17(6):734-40. doi:10.1093/humupd/dmr029

23. van der Linden M, Buckingham K, Farquhar C, Kremer JA, Metwally M. Luteal phase support for assisted reproduction cycles. Cochrane Database Syst $\operatorname{Rev}(2015)$ (7):CD009154. doi:10.1002/14651858.CD009154.pub3

24. Martins WP, Ferriani RA, Navarro PA, Nastri CO. GnRH agonist during luteal phase in women undergoing assisted reproductive techniques: systematic review and meta-analysis of randomized controlled trials. Ultrasound Obstet Gynecol (2016) 47:144-51. doi:10.1002/uog.14874

25. Isik AZ, Caglar GS, Soezen E, Akarsu C, Tuncay G, Ozbicer T, et al. Single dose $\mathrm{GnRH}$ agonist administration in the luteal phase of $\mathrm{GnRH}$ antagonist cycles: 
a prospective randomized study. Reprod Biomed Online (2009) 19(4):472-7. doi:10.1016/j.rbmo.2009.04.001

26. Pirard C, Loumaye E, Laurent P, Wyns C. Contribution to more patientfriendly ART treatment: efficacy of continuous low-dose GnRH agonist as the only luteal support-results of a prospective, randomized, comparative study. Int J Endocrinol (2015) 2015:727569. doi:10.1155/2015/727569

27. Bar Hava I, Blueshtein M, Hadas GH, Omer Y, Ben David G. Gonadotropinreleasing hormone analogue as sole luteal support in antagonist-based assisted reproductive technology cycles. Fertil Steril (2017) 107(1):130-5.e1. doi:10.1016/j.fertnstert.2016.10.011

28. Aboulghar MA, Marie H, Amin YM, Aboulghar MM, Nasr A, Serour GI, et al. GnRH agonist plus vaginal progesterone for luteal phase support in ICSI cycles: a randomized study. Reprod Biomed Online (2015) 30(1):52-6. doi:10.1016/j.rbmo.2014.09.017

29. Kung HF, Chen MJ, Guua HF, Chen YF, Yi YC, Yen-Ping Ho J, et al. Luteal phase support with decapeptyl improves pregnancy outcomes in intracytoplasmic sperm injection with higher basal follicle-stimulating hormone or lower mature oocytes. JChin Med Assoc (2014) 77:524-30. doi:10.1016/j. jcma.2014.07.001

30. Valbuena D, Martin J, de Pablo JL, Remohí J, Pellicer A, Simón C. Increasing levels of estradiol are deleterious to embryonic implantation because they directly affect the embryo. Fertil Steril (2001) 76:962-8. doi:10.1016/ S0015-0282(01)02018-0

31. Mitwally MF, Bhakoo HS, Crickard K, Sullivan MW, Batt RE, Yeh J. Estradiol production during controlled ovarian hyperstimulation correlates with treatment outcome in women undergoing in vitro fertilization-embryo transfer. Fertil Steril (2006) 86:588-96. doi:10.1016/j.fertnstert.2006. 02.086

32. Joo BS, Park SH, An BM, Kim KS, Moon SE, Moon HS. Serum estradiol levels during controlled ovarian hyperstimulation influence the pregnancy outcome of in vitro fertilization in a concentration-dependent manner. Fertil Steril (2010) 93:442-6. doi:10.1016/j.fertnstert.2009.02.066

33. Aktan E, Bozkurt K, Ozer D, Yucebilgin S, Karadadas N, Bilgin O. The effect of mid-luteal estradiol level on the outcome of ICSI-ET cycles. Arch Gynecol Obstet (2004) 269:134-8. doi:10.1007/s00404-0030533-6
34. Casper RF, Yen SS. Induction of luteolysis in the human with a long-acting analog of luteinizing hormone-releasing factor. Science (1979) 205:408-10. doi:10.1126/science.377491

35. Vicdan K, Zeki Isik A. Luteal phase hormonal profile in prediction of pregnancy outcome after assisted reproduction. Eur J Obstet Gynecol Reprod Biol (2001) 96:98-101. doi:10.1016/S0301-2115(00)00400-0

36. Fauser BC, Devroey P. Reproductive biology and IVF: ovarian stimulation and luteal phase consequences. Trends Endocrinol Metab (2003) 14:236-42. doi:10.1016/S1043-2760(03)00075-4

37. Hugues JN, Cedrin-Durnerin I, Bsandig B, et al. Administration of gonadotropin-releasing hormone agonist during the luteal phase of $\mathrm{GnRH}$-antagonist IVF cycles. Hum Reprod (2006) 21:article 13.

38. Humaidan P, Polyzos NP, Alsbjerg B, Erb K, Mikkelsen AL, Elbaek HO, et al. GnRHa trigger and individualized luteal phase hCG support according to ovarian response to stimulation: two prospective randomized controlled multi-centre studies in IVF patients. Hum Reprod (2013) 28(9):2511-21. doi:10.1093/humrep/det249

39. Daya S. Pitfalls in the design and analysis of efficacy trials in subfertility. Hum Reprod (2003) 18:1005-9. doi:10.1093/humrep/deg238

40. Vail A, Gardener E. Common statistical errors in the design and analysis of subfertility trials. Hum Reprod (2003) 18:1000-4. doi:10.1093/humrep/deg133

41. Griesinger G. Beware of the 'implantation rate'! Why the outcome parameter 'implantation rate' should be abandoned from infertility research. Hum Reprod (2016) 31(2):249-51. doi:10.1093/humrep/dev322

Conflict of Interest Statement: ABenmachiche, SB, AZ, and ABoularak have nothing to disclose. PH reports personal fees from Merck, MSD, and IBSA, grants from Merck, MSD, and Ferring, outside the submitted work.

Copyright $\odot 2017$ Benmachiche, Benbouhedja, Zoghmar, Boularak and Humaidan. This is an open-access article distributed under the terms of the Creative Commons Attribution License (CC BY). The use, distribution or reproduction in other forums is permitted, provided the original author(s) or licensor are credited and that the original publication in this journal is cited, in accordance with accepted academic practice. No use, distribution or reproduction is permitted which does not comply with these terms. 